\title{
Reflexión colectiva sistemática: un estudio orientado al desarrollo profesional docente
}

\author{
Jorge Catalán-Ahumada \\ Universidad de La Serena - La Serena - Chile \\ Pablo J. Castro \\ Universidad de La Serena - La Serena - Chile
}

\begin{abstract}
Resumen
Este trabajo expone el curso seguido por una intervención para desarrollar reflexión colectiva sistemática, en reuniones de profesores de un establecimiento público de educación media de La Serena, Chile y las posibilidades de cambio a partir de ella. Se utilizó una metodología cualitativa, donde se emplearon observaciones de reuniones de trabajo, entrevistas y bitácoras de investigadores. El análisis de datos consistió en síntesis descriptivas y análisis de contenido, con apoyo del programa computacional Atlas-Ti. Los resultados pusieron de manifiesto la potencialidad de la intervención para desarrollar reflexión colectiva sistemática; la apropiación que hicieron los profesores de sus teorías subjetivas, como también, las dificultades y requerimientos de la cultura escolar para realizar procesos de reflexión.
\end{abstract}

Palabras clave: Subjetividad; trabajo docente; desarrollo profesional.

\section{Collective systematic reflection:}

\section{an oriented teacher profesional development study}

\begin{abstract}
This study analyzed the course of an intervention aimed at developing a systematic collective reflection among educators of a vocational secondary educational establishment. The process of intervention was conducted during profesional meetings between educators, in which the discussions centered on their daily tasks, and the posible changes that may stem from such intervention. The design of the study here in is a case study of a process of intervention. While this study was descriptive-interpretative in nature and included substantive aspects aimed at altering teaching practices. Data was collected through observation of work meetings, interviews and investigator logs. Data analysis consisted of descriptive summaries and content an alysis, with the aid of the computer program Atlas-Ti. The results indicate that the process of intervention has prospects for developing a systematic collective reflection. Lastly, it is clear that there exist difficulties and requirements within the school setting in order to conduct collaborative reflection processes.
\end{abstract}

Keywords: Subjectivity; teachingwork; profesional development.

\section{Reflexão coletiva sistemática:}

\section{um estudo orientado para o desenvolvimento profissional docente}

\section{Resumo}

Este trabalho apresenta uma intervenção para o desenvolvimento de reflexão coletiva sistemática em reuniões de professores de uma escola pública de ensino médio de La Serena, Chile e as possibilidades de mudança a partir dela. Foi utilizada uma metodologia qualitativa, com observações de reuniões de trabalho, entrevistas e diários de bordo dos investigadores. A análise de dados consistiu em sínteses descritivas e análise de conteúdo, com apoio do programa computacional Atlas-Ti. Os resultados revelaram a potencialidade da intervenção para o desenvolvimento de reflexão coletiva sistemática; a apropriação que os professores fizeram de suas teorias subjetivas, como também as dificuldades e necessidades da cultura escolar para realizar processos de reflexão.

Palavras chave: Subjetividade; trabalho docente; desenvolvimento profissional. 


\section{Introducción}

Tanto los estudios acerca de la profesión de profesor, como la autonomía atribuida a un profesional y las implicancias de ésta en la toma de decisiones (Hargreaves, 1996/1998; Perrenoud, 2004/2007) confieren a la profesionalización del profesor especial relevancia como objetivo a cumplir para llevar a cabo procesos de reforma.

Si bien, en los últimos decenios, insistentemente se plantea que la reflexión es una condición necesaria del estatus profesional, hay quienes lo consideran difícilmente alcanzable (Kincheloe, 2001). Cabe, entonces, realizar un mayor esfuerzo por relevar y abordar con sistematicidad los procesos reflexivos de los profesores, para así "reinventar la profesión" (Montero, 2001, p. 81). Esto hará la diferencia entre una concepción del profesor como "técnico" o como "profesional" que, en tanto práctico reflexivo, genera conocimiento. La diferenciación implica superar la reflexión instrumental, propia de una orientación técnica, incorporando condicionantes personales, sociales o políticas, favoreciendo así, mediante una orientación práctica, lageneración de conocimiento profesional (Bolívar, 2004; Perrenoud, 2004/2007; Reyes\& Rodríguez, 2001; Rodríguez, 1995; Schön, 1993).

En este contexto, surge como problema de estudio la siguiente interrogante: ¿Cuál es el impacto de una intervención para desarrollar reflexión colectiva sistemática (RCS) en los procesos reflexivos que los profesores realizan en sus reuniones profesionales y en la utilización de estos procesos en el abordaje de problemáticas del quehacer pedagógico?

Subyace a la interrogante enunciada la pretensión de generación de conocimiento profesional y la contribución a la autonomía, como atributos inherentes a la reflexión (Álvarez \& cols., 2011; De Vicente, 2002; Montero, 2001).Planteado así el problema,

por reflexión colectiva sistemática se ha de entender la reflexión que realizan e intercambian los profesores, actuando como equipo de trabajo que ha recibido capacitación para analizar, intensificar y coordinar los procesos reflexivos en el abordaje de materias profesionales que les son relevantes (Catalán, 2011, p. 207).

La propuesta relaciona diversas concepciones teóricas y aspectos de política, normativa y funcionamiento del sistema educacional chileno. El objeto a investigar fueron los procesos reflexivos de los profesores(as) en situaciones de interacción entre pares, cuando abordan temáticas o problemáticas de su quehacer profesional.

El foco en estos procesos reflexivos fueron las teorías subjetivas (TS) sustentadas - advertida o inadvertidamente - por los profesores en sus reuniones de trabajo. El locus de los procesos reflexivos fue lo colectivo (Rodrigo, 1993) y no lo individual, asumiendo una perspectiva de creciente relevancia (Saussez, Ewen, \& Girard, 2001; Paquay, 2005; Saussez).
En lo que sigue, se analiza y fundamenta aspectos teóricos relacionados con el problema de investigación, desarrollando sucintamente una aproximación a los conceptos y teorías que sustentan el estudio.

\section{Antecedentes}

\section{El Paradigma del Pensamiento del Profesor}

El año 1975, la realización del Congreso del National Institute of Education, marca el inicio del llamado "paradigma del pensamiento del profesor", que concibe al profesor como un profesional reflexivo. El desarrollo del paradigma del pensamiento del profesor tuvo dos supuestos básicos: a) considerar a los profesores como profesionales que formulan juicios y toman decisiones y b) que su comportamiento se guía por pensamientos, juicios y decisiones (Shavelson \& Stern, 1989). Si bien estos supuestos constituyeron una base común, los estudios que se generaron siguieron cursos que dieron lugar a diversos "paradigmas y perspectivas de análisis” (Pradas, 2010, p. 24), poniendo en evidencia problemas relacionados con la consistencia o inconsistencia entre pensamiento y acción, imprecisiones conceptuales y limitaciones metodológicas para el acceso al objeto de estudio.

Probablemente, los principales cuestionamientos en los dos primeros decenios de desarrollo del paradigma del pensamiento del profesor provinieron de las expectativas cifradas en la reflexión para el cambio en el quehacer de los profesores.

Feldman (2001) cuestiona la continuidad del paradigma, atribuyendo su decadencia al reduccionismo descriptivo y a la asignación de un rol "revelador" a la reflexión en la práctica educativa. Al respecto, cabe señalar que si bien las problemáticas de investigación que involucran el pensamiento del profesor, especialmente en el último decenio, son abordados desde el enfoque denominado "conocimiento profesional del profesor", se mantiene y, aún más, se ha incrementado la preocupación por el papel preponderante de la reflexión en los procesos de cambio de los profesores (Arbeláez, 2007; Galaz, Fuentealba, Cornejo, \& Padilla, 2011; Kincheloe, 2001; Lessard, Altet, Paquay, \& Perrenoud, 2004; Marcelo, 2002; Marcelo \& Vaillant, 2009; Montero, 2001; Murillo, 2002).

Aun cuando no cabe soslayar los problemas conceptuales, teóricos y metodológicos advertidos en el paradigma del pensamiento del profesor, "reinventar la profesión" requiere la capacidad de tomar decisiones de los profesores, lo que se consigue al intensificar su propia indagación, a partir de dos consideraciones básicas: 1) el cambio no ocurre automáticamente por el mero hecho de reflexionar; y 2) para la resolución de los problemas del quehacer profesional, es indispensable la interacción entre el conocimiento que proviene de la reflexión individual o entre pares de profesores, con el conocimiento de la educación que proviene de la 
ciencia, en tanto constituyentes del llamado "conocimiento profesional” del profesor (Contreras, 2009; Marcelo, 2002; Montero, 2001).

\section{El desarrollo profesional del profesor}

La reflexión y, en este caso particular, la que tiene como objeto las teorías subjetivas de los profesores, tendría como principal aporte su contribución al desarrollo profesional; éste se ha conceptualizado de diversas formas, asociándolo a varios otros conceptos: perfeccionamiento de profesores, reciclaje de profesores, formación permanente, formación en servicio, desarrollo del profesorado. La más elemental y clara distinción parece ser la que diferencia el perfeccionamiento del desarrollo profesional por la intervención externa, en el primero; y por la dirección interna, en el segundo (De Vicente, 1992; Marcelo, 1999). El estudio que se reporta es más próximo a la segunda postura, orientándose al fortalecimiento de los procesos reflexivos de los profesores desde acciones realizadas por los propios profesores.

El desarrollo profesional parece utilizarse en dos sentidos básicos: uno se relaciona con la adquisición de conocimientos, destrezas y estrategias para procesar información y para desarrollar la enseñanza; el otro, con el cambio continuo que el desempeño profesional tiene en el tiempo, lo que permite establecer una progresión de etapas en algunos autores (De Vicente, 2002). En lo referente a la noción de perfeccionamiento, se reconocen varias tradiciones, que Zeichner (1993) agrupa en tres orientaciones básicas: la positivista, de mayor énfasis técnico; la interpretativa, que enfatiza lo cultural, naturalista; y la crítica, con interés en lo sociopolítico y emancipatorio.

Las orientaciones que han sustentado el desarrollo profesional de los docentes, o pragmáticas que han orientado el perfeccionamiento (Villar, 1991), permiten diferenciar tres modelos: uno se centra en la identificación y resolución de necesidades, otro centrado en la evaluación de la acción docente y uno centrado en la metáfora del profesor investigador, que concibe al profesor como un profesional reflexivo.

De Vicente (2002), en una revisión de los modelos y de autores que los tipifican, identifica tres aspectos básicos en la formación de los profesores: conocimiento, reflexión y colaboración. Subyace a estos aspectos la concepción del profesor como un profesional reflexivo, colaborador y que construye conocimiento. La reflexión se vincula estrechamente con la calidad educativa, ocupando un lugar central para promover el desarrollo e indispensable para conferirle poder y autonomía al profesor (De Vicente, 2002). La concepción del profesor como colaborador se sustenta en comprobaciones de que el pensamiento se construye interactiva, dialógica y argumentativamente; lo que justifica el trabajo escolar llevado a cabo por equipos de profesores (Hargreaves, 1996). Si bien la actividad profesional está estrechamente ligada con la actividad reflexiva, es una compleja conjunción de pensamientos, acciones, conocimientos, y emociones (Hargreaves, 2001); en tanto que la generación de conocimiento profesional es esencialmente colectiva y cooperativa, como también - de manera recomendable - de integración con otros equipos, conformando redes (Bolívar, 2010).

Junto con atribuirle al profesor un papel activo para guiar su propio trabajo, desde la práctica reflexiva se asume la capacidad del profesor para desarrollar el currículo y para promover los cambios que en la escuela se requieran (Arbeláez, 2007; Denegri, 2005). Estos cambios tienen como requerimiento esencial, contar con condiciones de gestión que lo favorezcan (Paquay, 2005); como al mismo tiempo, los cambios en los sujetos, específicamente en sus concepciones, crean conocimiento que lleva a cambiar las organizaciones (Marcelo, 2005; Nonaka \& Takeuchi, 1999).

La posibilidad de intervención de los profesores en aspectos que contribuyan al mejoramiento de la calidad de la educación, tiene como requerimiento que los propios profesores cambien y que este cambio surja de la evaluación de su quehacer y de lo que acontece en las instituciones en las que lo desarrollan (Cornejo, 2011; De Vicente, 1996; Murillo, 1999).

La práctica reflexiva debería sustentar la posibilidad de la evaluación del propio desempeño y ésta, a su vez, permitiría generar cambios individuales e institucionales; todo lo cual viene a justificar la relevancia del estudio realizado y sus proyecciones.

\section{Teorías Subjetivas}

Las teorías subjetivas son hipótesis que elaboran las personas para orientarse respecto de sí mismas y del mundo. Sirven a la vez como explicaciones, predicciones y prescripciones. Sirven para interpretar la realidad y como fundamento para actuar en ella (Catalán, 2010).El origen del concepto de "teoría subjetiva" es atribuido a Groeben y Scheele (1977, en Flick 1992), a propósito de la elaboración de una teoría de la construcción cognitiva de la realidad (Jancic Mogliacci, 2015), cuya estructura argumentativa admite una reconstrucción, a lo menos parcial, de un modo análogo a las teorías científicas.

Las teorías subjetivas cumplen diversas funciones: de orientación frente a situaciones específicas, justificación (o explicaciones a posteriori) y estabilización del sentimiento de propio valor (Flick, 1992). Otra función atribuida a las teorías subjetivas se refiere a su influencia en las relaciones intergrupos, como mediatizadoras de la imagen del mundo, favoreciendo un autoconcepto social (Rodríguez, 1993). Estos dos últimos, son aspectos de relevancia para el estudio que se reporta. Por una parte, de alguna manera, se esperaba la incidencia de la focalización en las teorías subjetivas en la cohesión y sinergia en el trabajo grupal; por otra, también se esperaba la incidencia en la autovaloración de los profesores en su condición profesional, por el reconocido impacto en el ejercicio del quehacer profesional (Voli, 1998; Miranda, Andrade, \& Freixas, 2001). 
En las teorías subjetivas, se puede distinguir una estructura superficial y una estructura profunda, que puede contener elementos implícitos y aun inconscientes; como también diversos grados de organización y formalización; desde formulaciones parcialmente explícitas, hasta redes de formulaciones (Catalán, 1997; Kelly, 1966). Botella (2001) ha llegado a establecer una analogía entre sistemas de construcciones personales y programas de investigación (en la concepción de Lakatos) o de paradigmas (en la concepción de Kuhn).

Sobre la base de una síntesis, integración y una progresiva elaboración teórica, se cuenta con una fundamentación y conceptualización de las teorías subjetivas que sustentan su valor heurístico y diferenciación con otros conceptos en uso, como también una propuesta para establecer propósitos y categorías de análisis para la investigación de las teorías subjetivas (Catalán, 2010).

\section{Objetivos}

El estudio se propuso: 1) Diseñar y aplicar un programa de capacitación en estrategias de reflexión colectiva sistemática a profesores de un establecimiento de educación media humanístico-científica de la IV Región de Coquimbo, Chile; 2) Describir, interpretar y contrastar los procesos reflexivos que ocurren en las sesiones de trabajo antes y durante la capacitación; 3) Analizar, según el juicio de los propios actores, los posibles cambios atribuibles a la intervención y traducibles en acciones observables.

\section{Diseño metodológico}

\section{Participantes}

La unidad de análisis - para la intervención - estuvo constituida por 33 profesores y profesoras de aula y de equipo de gestión de un establecimiento educacional técnico profesional de la comuna de La Serena, Chile. La muestra fue intencionada. Para la elección del establecimiento, se privilegió su carácter público (municipalizado), como opción por una modalidad de enseñanza en desventaja y que cumple con el marco regulatorio del Ministerio de Educación de Chile.

Como criterios de selección se consideró:

- Que no se encontraran participando en ninguna intervención sistemática o asesoría desarrollada por instituciones públicas o privadas, como una forma de reducir la influencia de variables ajenas a la investigación.

- Que las autoridades, manifestaran interés y se comprometieran a someterse a la intervención.
La elección de una muestra conformada por sólo un establecimiento para llevar a cabo la intervención obedeció a las siguientes consideraciones:

- La opción epistemológica asume la singularidad de una comunidad (establecimiento educacional), por lo que no se tiene pretensiones de generalización, ni se requiere representatividad estadística (cabe señalar que no se generalizará los resultados a otros educadores, aunque sí se pretende validar la intervención, la que podría ser replicable con otros sujetos y contextos).

- El requerimiento de validez de carácter cualitativo de alcanzar "descripciones densas", impone la realización de observaciones prolongadas en el tiempo (varios meses en las diversas fases) lo que haría inconveniente e innecesario seleccionar muestras más grandes en tamaño, en varios establecimientos, y altos costos, que podrían haber hecho inviable la ejecución del Proyecto.

\section{Capacitación}

Las estrategias metodológicas empleadas en la capacitación fueron las siguientes:

- A partir de exposiciones de los propios investigadores y de las demostraciones que realizaron, los profesores ejercitaron el reconocimiento de conceptos y estrategias, relacionados con TS, su identificación y análisis, como también con el curso y facilitación de procesos reflexivos.

- Utilizando como insumos los registros realizados por los investigadores en la fase "antes" de la intervención, los profesores analizaron las sesiones de trabajo en las que participaron, reconociendo obstáculos y elementos favorecedores para resolver las problemáticas de las sesiones correspondientes y proponiendo cursos de acción alternativos.

- La fase "durante la intervención" proporcionó nuevos materiales de análisis, de dos formas básicas: a) situaciones emergentes en que aparecían conceptos, estrategias y condiciones favorecedoras $u$ obstaculizadoras, que se esperaba que los propios profesores podrían identificar; b) análisis propuestos por los docentes a cargo, a partir de registros en bitácoras (realizados por los investigadores) del proceso mismo de intervención.

- A los profesores se le proponían situaciones o casos a analizar, en forma individual o colectiva, semanalmente. 
Procedimientos empleados para la Recolección de datos

Los recursos utilizados para la recolección de datos fueron los siguientes:

a) Observaciones con registro de audio (y algunas filmaciones) y notas de campo de las sesiones de trabajo de grupo de profesores (as), "antes" y "durante" Las observaciones para la fase "antes" se realizaron en sesiones semanales, por tres meses. Las observaciones para la fase "durante" se realizaron en sesiones semanales por cuatro meses. El registro se centró en el curso que tienen los procesos de reflexión y, más específicamente, el uso de teorías subjetivas. Se complementó con observaciones de condiciones de gestión facilitadoras u obstaculizadoras de la reflexión.

b) Bitácoras que llevaron los miembros del equipo de investigación en la fase "durante" la intervención, acerca de sus vivencias, impresiones e interpretaciones.

c) Realización de 4 entrevistas semiestructuradas, a informantes clave.

d) Reportes escritos de todos los profesores participantes del establecimiento, con sus juicios acerca de la intervención (correspondientes a la fase "después").

\section{Análisis de datos}

Los procedimientos de análisis de datos fueron los siguientes:

a) Los datos correspondientes se sometieron a síntesis descriptivas y a análisis de contenido, con el apoyo del programa computacional Atlas-Ti, siguiendo categorizaciones de la grounded theory (Strauss \& Corbin, 1991).

b) Luego de un análisis independiente, se procedió a la contrastación de los resultados del "antes" y "durante". Las entrevistas también se sometieron a análisis de contenido con Atlas-Ti.

\section{Resultados}

\section{Indagaciones Etapa "Antes"}

Primera indagación: El análisis de la fase "antes" permite organizar los datos en dos momentos del proceso reflexivo.
El primer momento está relacionado con el análisis de situaciones problemáticas del quehacer cotidiano de los profesores, manifestadas en sus sesiones de trabajo. En este momento, se enuncian los problemas, en general, atribuyéndolos a factores externos al profesorado, predominando teorías subjetivas "inhibidoras de acción”, cuya argumentación llevar a concluir, explícita o implícitamente que hay poco o nada que hacer, por tratarse de causales relacionadas con la familia, el entorno (como la influencia de "líderes negativos"), problemas de las propias estudiantes, etc., en todos los cuales la intervención de la escuela no es posible.

Un segundo momento reflexivo, lleva a los docentes a buscar algún tipo de participación, ya sea como paliativo o como aporte a la solución de los problemas de las estudiantes. En especial, concuerdan y proponen crear condiciones para el aprendizaje y un abordaje más colectivo de la problemática. Se verifica voluntad para contribuir a las soluciones. En esta fase, las teorías subjetivas sustentadas por los profesores se pueden considerar "iniciadoras de acción", es decir, constituyen argumentaciones que promueven, o dan cabida a la realización de acciones para superar la problemática a la que hacen referencia.

Segunda indagación: Basados en las observaciones de las reuniones de trabajo de los profesores, antes de la intervención, se realizó un análisis específico de éstas con el interés de describir e interpretar las teorías subjetivas de este grupo de profesores acerca de la motivación escolar (rol como agente motivacional y motivación inherente a las alumnas) y sus expectativas acerca del éxito y fracaso escolar (Brighenti Bortoluzzi \& Catalán, 2014).

Los datos recolectados mediante la observación directa se complementaron con el análisis de una entrevista grupal que formaba parte de otro estudio realizado en el establecimiento (Retuert \& Castro, no prelo). Los resultados mostraron que la sobre atribución que hacen los profesores acerca de su responsabilidad como agente motivacional, ignora otras variables dependientes tanto de las alumnas como del contexto educativo. Además, que sus expectativas de éxito o fracaso están centradas en la realización de la práctica y posterior obtención de la especialidad técnica. Ignorando con esto, los intereses de las alumnas que están más motivadas hacia la continuación de sus estudios (Castro-Carrasco \& cols., 2012).

\section{Indagación etapa "durante"}

En esta etapa se realizaron observaciones, registro en bitácoras y entrevistas. El objetivo fue describir y comprender las variables que, desde la perspectiva de los profesores y la de los investigadores, se relacionaban con la disposición a la reflexión profesional, durante esta capacitación. Los resultados muestran que sus Teorías Subjetivas incluyen distintos tópicos relacionados con la disposición ante la RCS, incluyéndose factores, o atribuciones de control, que facilitan o dificultan el proceso, de tipo "interno y 
externo". Factores como la influencia negativa del horario en que se realizaban las sesiones de trabajo y la percepción general de contar con Escasas instancias para la reflexión, operan como externos. Factores internos son su motivación diversa por la reflexión colectiva sistemática y la buena relación entre ellos como comunidad (Castro, Agüero, Barraza, Escobar, \& Jorquera, 2012).

\section{Indagación Etapa "Después"}

En general, los juicios emitidos por los y las docentes acerca de la intervención y del proceso seguido ponen en evidencia la valoración de la reflexión en el quehacer cotidiano del profesor y de sus implicancias en el desarrollo profesional: "En este ambiente propicio al debate aparecieron opiniones que no siempre encuentran espacio a la discusión" (profesora 3)

Recurrentemente se menciona la falta de tiempo para reflexionar, como igualmente la hora de la tarde de la actividad (siempre se realizó en el horario de reuniones y éste correspondía a la última hora de clases).

Hubo algunas observaciones acerca de que la actividad se refería a temáticas que no eran de interés de los profesores. Al respecto, cabe señalar que las temáticas para la reflexión colectiva sistemática estaban tomadas de las sesiones de los propios profesores, en sus propias reuniones.

Hay muchos comentarios que hacen referencia a la importancia y aporte de reflexionar, como de ciertas condiciones necesarias para llevarla a cabo: “... Por lo tanto, se deben generar los espacios y tiempos adecuados para desarrollar jornadas de reflexión pertinentes en el establecimiento y de esa manera aplicar los resultados obtenidos..." (profesora 2).

Por otra parte, se advierte niveles diversos de toma de conciencia del sentido de la reflexión realizada:

La intervención en una primera instancia nos pareció ambigua, poco clara en relación a otras instancias de perfeccionamiento, tal vez porque el desarrollo de este taller tenía como finalidad el autoanálisis interno, personal e institucional y por tal razón éramos nosotros los llamados a generar nuestra propia estructura de reflexión, para eso era necesaria la observación de nuestra labor por los profesionales a cargo (profesora 8).

También hay diversidad de grados de compromiso asumido, de logros y de participación en procesos reflexivos:

Falta de compromiso de los colegas para realizar los trabajos. Esta reflexión no ayudó para mejorar nuestra labor docente. (profesora 1).

... uno de los aspectos negativos de estas reflexiones es que no siempre todos los y las docentes participan efectivamente y algunos se abstienen de hacerlo por temores mal fundados y por otras diversas razones. (profesora 4).
Creo que hay mucho que mejorar, partiendo de la base que las reuniones técnicas deberían ser una instancia de reflexión y pienso que no es así. (profesora 11).

En síntesis, los juicios emitidos por profesoras y profesores ponen en evidencia un hacerse cargo de la relevancia de los procesos reflexivos en el abordaje de los problemas del quehacer pedagógico cotidiano, dificultades internas para llevarlos a cabo y algunas resistencias para hacerlo con intervención externa (de los investigadores). Hay comentarios reveladores de autocrítica a las dificultades para actuar como colectivo y una actitud proactiva manifestada en la verbalización del interés por realizar más reflexión y de mayor profundidad.

\section{Discusión}

En esta investigación se diseñó y aplicó un programa de sesiones de trabajo con los docentes, basado en estrategias de reflexión colectiva sistemática. Este programa diseñado a partir del trabajo con las teorías subjetivas de los propios docentes, recolectadas durante la etapa antes, permitió dar pertinencia al contenido de las sesiones de trabajo. Sin embargo, es interesante advertir que a pesar de aquello, algunos docentes calificaron el contenido de las sesiones como ajeno a sus intereses. Esto lo interpretamos en dos direcciones.

Por una parte, podría existir una hegemonía de tópicos y opiniones expresadas en las reuniones, ejercidas por las autoridades del establecimiento o por aquellos profesores más expresivos, que no representan a todos los docentes. Por otra parte, es posible que si bien los profesores manifiesten, de manera más o menos explícita, teorías subjetivas acerca de determinados temas, estos temas les resulten ya poco motivadores para ser reflexionados, quizá por la orientación negativa y de inhibición de la acción que suelen contener, esta última característica de las teorías subjetivas de grupos de profesores, se ha encontrado en otros estudios realizados en contextos educativos similares (véase, por ejemplo, Cuadra, Jorquera, \& Pérez, 2015).

Los procesos reflexivos que ocurrieron durante la capacitación se caracterizaron por la identificación de dificultades externas e internas al proceso de reflexionar propiamente tal, como a su quehacer en general. Esto viene a confirmar el planteamiento teórico de la construcción interactiva, dialógica y argumentativa del conocimiento profesional de profesores (Hargreaves, 1996).

Temas como la atribución externa a la familia u origen social de las alumnas como causas del fracaso escolar o desmotivación de las estudiantes, fueron recurrentes. Esto es compatible con numerosos estudios que muestran como existe una tendencia en los docentes a percibir su quehacer como controlado externamente, ya sea por condiciones de los estudiantes o por regulaciones del sistema escolar (Cuadra, 2009; Volante \& Müller, 2006). 
Cabe hacer notar, que lo anterior contrasta parcialmente con lo observado en la etapa antes de indagación, en donde se observó un segundo momento reflexivo en el que los profesores buscan soluciones propias, internas y controlables, a las dificultades detectadas. Dicha tendencia, no observada con claridad durante las sesiones de trabajo de implementación de la estrategia, podrían explicarse por un efecto no deseado de la estrategia, de estructuración del trabajo de los docentes, situándose ellos prioritariamente en una etapa de diagnóstico de los problemas.

Según los juicios observados y expuestos aquí de los docentes, podrían atribuirse a la intervención cambios relativos a una consolidación de la conciencia de la importancia de contar con procesos reflexivos sistemáticos y con tiempos adecuados para ello. Los docentes no emiten juicios respecto a cambios en sus acciones. Esto es coherente con lo anterior, en el sentido que la mayor parte de sus procesos reflexivos durante la capacitación estuvieron marcados por la identificación de problemas y de sus explicaciones asociados a ellos. Cuando se realizaron actividades tendientes a propiciar la generación de soluciones se observó alguna resistencia y tendencia a pensar en soluciones clásicas, repetitivas, y representativas de su modo tradicional de abordar los problemas.

Como limitación al diseño empleado, observamos la falta de un trabajo dialógico más personalizado, complementario a la intervención colectiva, el que bien pudo lograrse con más entrevistas en profundidad en la fase "durante". Otra limitante en la estrategia utilizada, pudo ser la probable excesiva confianza por parte de los investigadores/ facilitadores respecto a la potencialidad de trabajar con los docentes sobre los contenidos de sus propias teorías subjetivas, recogidos en la etapa "antes"; podemos hipotetizar que exponerles a los docentes sus propias creencias, podría haber generado resistencia en ellos e inhibirse así actitudinalmente ante esta intervención. Equilibrar la generación de confianza en los facilitadores, sin sacrificar el rol que tienen de confrontar a los participantes y generar una cierta "incomodidad productiva" o generadora de cambio, es un desafío reconocido (Vanassche \& Kelchtermans, 2015).

En esta intervención lo anterior pudo ocurrir al confrontar a los profesores con sus propias teorías subjetivas, generando el posible distanciamiento actitudinal de ellos hacia la intervención. Alguna evidencia de esta hipótesis fue posible tener durante el proceso de capacitación. Adicionalmente, y pensando en futuras intervenciones, convendría reevaluar la combinación de técnicas de reflexión oral versus escrita, esto a la luz de estudios que muestran diferencias en ellas de acuerdo a los propósitos que se quieren lograr; centramiento en razonamiento narrativo práctico o bien teórico (subjetivo, en nuestro enfoque) generador de conocimiento práctico. Lo anterior, junto con evaluar la inclusión de facilitadores con experiencia escolar cercana que guien las reflexiones orales que se realizan, para el logro de la revisión de su conocimiento práctico por parte de los docentes a partir del análisis de sus teorías subjetivas, en concordancia con los hallazgos de Leijena y cols. (2015).
El análisis del proceso de la intervención en sus diversas fases lleva a los investigadores a plantear que las proyecciones del estudio se orientan a optimizar las condiciones de gestión educacional, como un requerimiento indispensable para desarrollar reflexión colectiva sistemática y favorecer así, el fortalecimiento de la profesionalidad docente. La gestión educacional la entendemos como la manera que tiene la institución educacional de organizarse, de acuerdo a su cultura, clima institucional, tipo de relaciones y modos de comunicación; de modo que actúa como factor de contexto de la enseñanza y particularmente de las prácticas pedagógicas de los profesores. Lo anterior desde una visión de desarrollo profesional que, junto con destacar la importancia de la reflexión docente, asume la importancia de un cambio colectivo y organizacional por sobre el individual (Bolívar, 2010; Smith, Hofer, Gillespie, Solomon, \& Rowe, 2009).

Específicamente, dos variables podrían relacionarse estrechamente con el ejercicio de la reflexión profesional colectiva, ya sea como facilitadoras u obstaculizadoras, además de constituir factores significativos en la mejora escolar (Arzola, Vizcarra, \& Zabalza, 2002): liderazgo educativo y visión institucional compartida.

En lo que concierne al liderazgo, parece promisorio explorar la potencialidad del "liderazgo profesoral":

Aquellos... [los líderes profesorales] son reflexivos, inquisitivos, centrados en mejorar su oficio, y orientados hacia la acción; aceptan la responsabilidad que implica la enseñanza de alumnos, y tienen un fuerte sentido de los que representan... están dispuestos a aprender, y entienden las tres dimensiones de la educación escolar: enseñanza de los alumnos, de los colegas, y de sí mismos (Lambert, 2003, p. 2, traducción nuestra).

En cuanto a la visión institucional compartida, nos parece clave considerar la influencia del grado en que los profesores comparten o no propósitos comunes definidos por ellos mismos, y por tanto, su capacidad como colectivo de ir generando compromisos con el logro de éstos (Manterola \& Astudillo, 2002).

Lo que en términos organizacionales se denomina visión institucional, supone la presencia de elementos aglutinadores de la acción, que en las organizaciones educativas se expresa en creencias centrales que responden efectivamente a las necesidades de todos, en un contexto en donde las contribuciones de los miembros de la comunidad educativa a esta visión son reconocidas. Esto implica además, a nivel de gestión, procesos de identificación y clarificación de los obstáculos para el logro de esta visión (Interstate School Leaders Licensure Consortium, 1996).

La existencia de una visión compartida supone además la presencia de un proyecto educativo común al interior de la institución (Villa \& Yániz, 1999), sin embargo nos parece importante considerar la posibilidad permanente de construcción, o de reconstrucción, de dicha visión, por tanto la necesaria comunalidad no implica la imposibilidad 
de disenso, es decir, de procesos de "negociación" entre los profesores, a través de la reflexión colectiva, de los objetivos comunes a definir.

Como afirmó Senge, hace más de 20 años atrás, la práctica de la visión compartida propicia el compromiso genuino (Senge, 1992). Podemos hipotetizar que la experiencia extraída en la intervención muestra cómo aun lo que es constitutivo del propio quehacer cotidiano, se puede considerar ajeno cuando los docentes lo asumen como impuesto o surgido de decisiones de las que no se sienten partícipes.

De lo que se trata, consecuentemente, es de promover el compromiso de los profesores; pero no como una tarea individual, sino como una indispensable y larga tarea colectiva que se manifieste en la cultura organizativa de la escuela (Bolívar, 2010).

\section{Conclusiones}

El análisis interpretativo de los resultados lleva a concluir que: 1) la capacitación en reflexión colectiva sistemática permite dar cuenta del curso de los procesos reflexivos de los profesores para analizar problemáticas de su propio quehacer, caracterizados por una primera atribución externa para luego reconocer implicancias internas y la búsqueda tentativa de soluciones; 2) los profesores valoran la práctica de la reflexión colectiva, a la vez que reconocen su propia toma de conciencia de problemáticas institucionales mediante procesos reflexivos; 3) resistencias a la intervención externa para abordar problemas del establecimiento que, dadas limitaciones de gestión internos - traducibles en tiempos muy acotados de reflexión, horarios inadecuados para realizarlos y un contexto de excesivas exigencias administrativas - reducen las posibilidades de reflexión de mayor profundidad, alcance y de búsqueda de soluciones alternativas y de mayor elaboración; 4) el cambio en las teorías subjetivas, producto de la reflexión colectiva sistemática no tiene una traducción inmediata en el comportamiento.

El análisis de las conclusiones extraídas permite sustentar, a la luz de los antecedentes teóricos del presente estudio, la potencialidad de la reflexión colectiva sistemática para promover el desarrollo profesional de los docentes, en el entendido que tanto el cambio en las teorías subjetivas como el cambio en el comportamiento del profesorado de un establecimiento educacional, puede y debe contribuir al cambio en la cultura organizacional, de modo de avanzar hacia una visión básica compartida y al ejercicio del quehacer profesional con mayor autonomía.

\section{Referencias}

Álvarez, F. Abrahams, M.J., Gaete, C. Galdames, V., Latorre, M., Moira Lee, M., \& Rojas, M.T. (2011). Saber pedagógico y formación inicial de docentes. Recuperado: 19 jan. 2015. Disponível: http:// www.ceppe.cl/images/stories/recursos/publicaciones/Marisol\%20 Latorre/SABER-PEDAGOGICO-Y-FORMACION-INICIAL-DE-

\section{DOCENTES1.pdf}

Arbeláez, R. (2007). En el reconocimiento de las concepciones docentes se encuentra el camino del mejoramiento continuo de la calidad docente. Recuperado: 19 jan. 2015. Disponible:http://www. uv.es/arbelaez/v2n210reconocimientodeconcepciones.htm

Arzola, S., Vizcarra, R., \& Zabalza, J. (2002). Autoevaluación de la gestión escolar: propuesta de un modelo. Boletín de Investigación Educacional, 17 (1), 53-81.

Bolívar, A. (2004). El conocimiento de la enseñanza: explicar, comprender y transformar. Salusvita, 25(1), 17 - 42.

Bolívar, A. (2010). La lógica del compromiso del profesorado y la responsabilidad del centro escolar. Una revisión actual. Revista Iberoamericana sobre Calidad, Eficacia y Cambio en Educación, 8 (2). Disponível: http://www.rinace.net/reice/numeros/arts/ vol8num2/art1

Botella, L. (2001). El ser humano como constructor de conocimiento: el desarrollo de las teorías científicas y las teorías personales. Recuperado: 19 jan. 2015. Disponível: http://padron.entretemas. com/cursos/deteorias/LECTURAS/Botella_Constructor $\% 20$ del\%20conocim.htm

Brighenti Bortoluzzi, M. \& Catalán, J. (2014) Teorías subjetivas de profesores en reuniones de trabajo: un estudio descriptivointerpretativo. Psicologia Escolar e Educacional, 18 (1), 151-159. Disponível: http://dx.doi.org/10.1590/S1413-85572014000100016

Castro-Carrasco, P., General, F., Jofré, R., Sáez, N., Vega, Á., \& Bortoluzzi, M. (2012). Teorías subjetivas de profesores sobre la motivación y sus expectativas de éxito y fracaso escolar. Educar em Revista, 46 (4), 159-172.

Castro, P., Agüero, C., Barraza, A., Escobar, G. \&Jorquera, J. (2012). Disposición a la reflexión colectiva sistemática en docentes de un centro educativo de Chile. Revista Mexicana de Investigación Educativa, 17 (53), 573-591.

Catalán, J. (1997). La profesión del profesor según las teorías subjetivas de los formadores. Tesis de Doctorado, Pontificia Universidad Católica de Chile, Santiago, Chile.

Catalán, J. (2010). Teorías subjetivas. Aspectos teóricos y prácticos. La Serena: Editorial Universidad de La Serena.

Catalán, J. (2011). Del pensamiento al conocimiento profesional del profesor. Em J. Catalán (Org.), Psicología Educacional. Rumbos, problemáticas y aportaciones (pp. 187- 215). La Serena: Editorial Universidad de La Serena.

Contreras, L. (2009). Concepciones, creencias y conocimiento: Referentes de la práctica profesional. Revista Electrónica Iberoamericana de Educación en Ciencias y Tecnología, 1 (1), 11-36. Recuperado: 19 jan. 2015. Disponível:http://www. 
exactas.unca.edu.ar/riecyt/VOL\%201\%20NUM\%201/Doc\%20 RIECyT\%201-1.pdf

Cornejo, J. (2011). El self study de la práctica de los formadores: bases teóricas, características y modalidades metodológicas. Em A. Galaz, R. Fuentealba, J. Cornejo, \& A. Padilla (Orgs.), Estrategias reflexivas en la formación de profesores y de formadores de profesores. ¿Qué desafíos se proyectan desde la formación basada en competencias? (pp. 115-174). Santiago de Chile: Gráfica LOM,

Cuadra, D. (2009). Teorías subjetivas en docentes de una escuela de bajo rendimiento, sobre la enseñanza y el aprendizaje del alumno. Revista Mexicana de Investigación Educativa, 14 (42), 939-967.

Cuadra, D., Jorquera, R., \& Pérez, M.A. (2015).Las teorías subjetivas del profesor acerca de su salud laboral: Implicancias en la promoción de la salud preventiva en el trabajo docente. Ciencia \& trabajo, 17 (52), 1-6.

Denegri, M. (2005). Proyectos de aula interdisciplinarios y reprofesionalizacion de profesores: un modelo de capacitación. Estudios Pedagógicos, 31 (1), 33-50.

De Vicente, P. (1992). La adquisición del conocimiento pedagógico del contenido por una profesora principiante. Em A. Estebaranz \& V. Sánchez (Orgs.), Pensamiento de los profesores y desarrollo profesional. Conocimiento y teorías implícitas (Tomo 1, pp. 183198). Sevilla: Secretariado de Publicaciones de la Universidad.

De Vicente, P. (1996). Formación y evaluación basada en el centro. Em Villa, A. (Org.), Evaluación de experiencias y tendencias en la formación del profesorado (pp. 289-232). Bilbao: ICE de la Universidad de Deuto.

De Vicente, P. (2002) (Org.). Desarrollo profesional del docente en un modelo colaborativo de evaluación. Bilbao: ICE, Universidad de Deusto.

Feldman, D. (2001). ¿Nuevos fundamentos para la práctica educativa y el curriculum? El caso de los estudios sobre el pensamiento del profesor. Em Associação Nacional de Pós-Graduação e Pesquisa em Educação (Org.),24a Reunião Anual da Anped.Caxambu, Brasil: Anped.

Flick, U. (1992). (Org.). La perceptionquotidienne de la santé et de la maladie. Théoriessubjectives et repréntations sociales. Paris: L'Hartmann.

Galaz, A., Fuentealba, R., Cornejo, J., \& Padilla, A. (Orgs.) (2011). Estrategias reflexivas en la formación de profesores y de formadores de profesores. ¿Qué desafíos se proyectan desde la formación basada en competencias? Santiago de Chile: Gráfica LOM.

Hargreaves, A. (1998). Profesorado, cultura y postmodernidad. Madrid: Morata. (Trabajo original publicado en 1996).
Hargreaves, A. (2001). Aprender a cambiar. Más allá de los objetivos. Madrid: Octaedro.

Jancic Mogliacci, R. (2015). Teachers' capability-related subjective theories in teaching and learning relations. Tesis de doctorado, Universidad de Bielefeld, Alemania. Disponível: http://pub.unibielefeld.de/publication/2730720

Interstate School Leaders Licensure Consortium. (1996). Standards for school leaders. Em M. Fullan (Org.), The Jossey-Bass reader on educational leadership (pp 97-113). San Francisco: JosseyBass.

Kelly, G. (1966). Teoría de la personalidad. La psicología de las construcciones personales. Buenos Aires: Troquel.

Kincheloe, J. (2001). Hacia una revisión crítica del pensamiento docente. Barcelona: Octaedro.

Lambert, L. (2003). Teachers as leaders: The heart of the high leadership capacity school (Los profesores en calidad de líderes son el centro de la escuela de alta capacidad de liderazgom versión traducida al español). Em L. Lambert, Leadership Capacity for Lasting School Improvement. Alexandria, VA: Association for Supervision and Curriculum Development.

Leijena, Ä., Allasa, R., Pedastea, M., Knezice, D., Mena, J., Meijerf, P., Husuc, J., Krulla, E., \& Toom, A. (2015). How to support the development of teachers' practical knowledge: Comparing different conditions. Procedia - Social and Behavioral Sciences191, 1205 $-1212$.

Lessard, C., Altet, M., Paquay, L., \& Perrenoud, P. (2004). Entre sens commun et sciences humaines. Quels savoirs pour enseigner. Bruxelles: De Boeck y Larcier, S.

Manterola, M., \& Astudillo, O. (2002). De una escuela que enseña a una escuela que aprende: el aprendizaje de los educadores. Boletín de Investigación Educacional, 17 (1), 82-100.

Marcelo, C. (1999). Aproximación al concepto desarrollo profesional de los profesores Em C. Marcelo (Org.), Formación del profesorado para el cambio educativo. Barcelona: Ediciones Universitarias de Barcelona.

Marcelo, C. (2002). La formación inicial y permanente del profesorado en España. Los cambios necesarios [Conferencia]. La profesión docente: situación actual y perspectivas. Actas de las Jornadas de reflexión y debate. Ciudad Real: Consejo Escolar Castilla-La Mancha.

Marcelo, C. (2005). La formación en tiempos de cambio: el principal problema y la mejor de las soluciones. Revista de empleo, 9 (1), $49-52$.

Marcelo, C., \& Vaillant, D. (2009). Desarrollo profesional docente ¿Cómo se aprende a enseñar? Madrid: Narcea. 
Miranda, C., Andrade, M., \& Freixas, G. (2001). Revalidación de constructo del Test de Autoestima en Profesores de Arzola. Boletín de Investigación Educacional, 16 (1), 105-120.

Montero, L. (2001). La construcción del conocimiento profesional docente, Santa Fe: Homo Sapiens Ediciones.

Murillo, P. (1999). El aprendizaje del profesorado y los procesos de cambio. Sevilla: Mergabulum Edición y Comunicación, S.L.

Murillo, P. (2002). Educación, sociedad y conocimiento: el peso de la realidad en el futuro de la formación. Revista PerspectivaCEP,4 (1), $93-109$

Nonaka, I. \& Takeuchi, H. (1999). La organización creadora de conocimiento. Cómo las compañías japonesas crean la dinámica de la innovación (M.H. Kocha,Trad.). México: Oxford University Press.

Paquay, L. (2005). Devenir des enseignants et formateurs professionnels dans une Organization apprenante? De l'utopie à la réalité!. European Journal of Teacher Education, 28(2), 111-128.

Perrenoud, P. (2007). Desarrollar la práctica reflexiva en el oficio de enseñar( $4^{\mathrm{a}}$ ed.). Barcelona: GRAÓ. (Trabajo original publicado en 2004).

Pradas, R. (2010). El estudio del pensamiento del profesorado sobre la toma de decisiones interactivas: análisis de un caso en educación física escolar. Cultura y Educación, 22 (1), 21-36.

Retuert, G. \& Castro, P.J. (no prelo). Teorías subjetivas de profesores acerca de su rol en la construcción de la convivencia escolar. Coquimbo: Universidad de La Serena, Magíster en Psicología.

Reyes, M. \& Rodríguez, J.M. (Orgs.) (2001). Estrategias para la formación del profesorado. Estudios y experiencias. Huelva: XYZ Ediciones.

Rodrigo, M.J. (1993). Representaciones y procesos en las teorías implícitas. Em M. J. Rodrigo, A. Rodríguez, \& J. Marrero (Orgs.), Las teorías implícitas. Una aproximación al conocimiento cotidiano (pp. 95-122). Madrid: Visor.

Rodríguez, A. (1993). El conflicto intergrupal desde las teorías implícitas. Em M. J. Rodrigo, A. Rodríguez \& J. Marrero (Orgs.), Las teorías implícitas. Una aproximación al conocimiento cotidiano(pp309-338). Madrid: Visor.

Rodríguez, J.M. (1995). Formación de profesores y prácticas de enseñanza. Huelva. Publicaciones Universidad de Huelva.

Saussez, F., Ewen, N., \& Girard, J. (2001). Au cœur de la pratique réflexive, la conceptualisation? Réflexions à partir d'um dispositif de formation au Grand-Duché de Luxembourg. Recherche et Formation, 36 (1), 69-87.
Schön, D. (1993). La formación de profesionales reflexivos. Barcelona: Paidós.

Senge, P. (1992). La quinta disciplina en la práctica. El arte y la práctica de la organización abierta al aprendizaje. Buenos Aires: Granica.

Shavelson, R., \& Stern, P. (1989). Investigación sobre el pensamiento pedagógico del profesor, sus juicios, decisiones y conductas. Em G. Sacristán, J. \& A. Pérez Gómez (Orgs.), La enseñanza. Su teoría y su práctica (pp. 372-419). Madrid: Akal.

Smith, C., Hofer, J., Gillespie, M., Solomon, M., \& Rowe, K. (2009). How teachers change. A study of profesional development in adult education. New York: Nova Science Publishers.

Strauss, A. \& Corbin, J. (1991). Basics of qualitative research. Grounded Theory procedures and techniques. Newbury Park: Sage Publications, Inc. Fourth Printing.

Vanassche, E. \& Kelchtermans, G. (2015). Facilitating self-study of teacher education practices: toward a pedagogy of teacher educator profesional development. Professional Development in Education.

Villa, A., \& Yániz, C. (1999). Aprendizaje organizativo y desarrollo profesional. Revista de currículum y formación del profesorado, $3(1), 1-20$

Villar, L.M. (1991). Paradigmas de formación del profesorado. Em A. Medina \& M.L. Sevillano (Orgs.), Didáctica (pp. 499-526). Madrid: UNED.

Volante, P. \& Müller, M. (2006). Cambios en el discurso sobre resultados de aprendizajes en escuelas de bajo rendimiento y alta vulnerabilidad social. Revista Pensamiento Educativo, 39(2), 205224.

Voli, F. (1998). La autoestima del profesor. Madrid: Editorial y Distribuidora S. A.

Zeichner, K. (1993). El maestro como profesional reflexivo. Cuadernos Pedagogía, 1 (220), 44-49. 


\section{Sobre os autores}

Jorge Catalán-Ahumada (jcatalan@userena.cl)

Doctor en Ciencias de la Educación, académico de la Universidad de La Serena, Chile.

Pablo J. Castro (pablocastro@userena.cl)

Doctor en Psicología, académico de la Universidad de La Serena, Chile.

Este artículo es inédito. El proyecto fue financiado por DIULS/ Universidad de La Serena. El presente reporte de investigación se enmarca en una investigación financiada por la Dirección de Investigación (DIULS) de la Universidad de La Serena, Proyecto DIULS PR0821. 
